\title{
Seasonal inhibition of puberty in domestic gilts is overcome by melatonin administered orally, but not by implant
}

\author{
A. M. Paterson ${ }^{1}$, C. A. Maxwell ${ }^{2}$ and A. Foldes ${ }^{2}$ \\ ${ }^{1}$ Animal Production Division, Department of Agriculture, South Perth, WA 6151, Australia; and \\ ${ }^{2}$ CSIRO Division of Animal Production, PO Box 239, Blacktown, NSW 2148, Australia
}

Summary. The ability of exogenous melatonin, applied either orally or by implant, to overcome the seasonal inhibition of puberty in domestic gilts was tested in two experiments. In Expt 1, 24 gilts received two melatonin implants at 126 days of age and again at 161 days and 196 days, while 24 gilts acted as controls. All gilts were slaughtered at a mean age of 223 days. Blood samples were collected by venepuncture from eight gilts in each treatment at 126, 144 and 178 days of age and the plasma was assayed for melatonin concentration by direct radioimmunoassay. In Expt 2A, four gilts ( 125 days of age) were fed either $0,1,2$ or $4 \mathrm{mg}$ of melatonin at 14:00 h on each of four consecutive days. Blood samples for melatonin assay were collected via indwelling jugular catheters every 30 or $60 \mathrm{~min}$ from 12:00 to 22:00 h. In Expt 2B, 27 gilts were fed $1 \mathrm{mg}$ of melatonin at 15:00 h each day from 129 days of age until slaughter at 221 days, while 25 gilts acted as controls. In both experiments, the presence of morphologically normal corpora lutea at slaughter was the criterion for puberty.

In Expt 1, constant-release melatonin implants had no effect on the percentage of gilts which reached puberty. Among the 24 control gilts, two $(8 \cdot 3 \%)$ reached puberty compared with one of the $24(4 \cdot 2 \%)$ gilts with implants. In all the samples from control gilts, and in the samples taken from treated gilts prior to implantation at 126 days of age, mean plasma melatonin concentration was below the sensitivity of the assay $(3.6 \mathrm{pg} / \mathrm{ml})$. In the samples taken from treated gilts 18 days after the first implants, mean plasma melatonin concentration had increased to $34.1 \mathrm{pg} / \mathrm{ml}(P<0.001)$. In the samples taken 17 days after the insertion of the second pair of implants, mean plasma melatonin concentration was higher than it had been at 144 days $(66.6 \mathrm{vs} .34 .1 \mathrm{pg} / \mathrm{ml}$, s.e.m. $=2 \cdot 03, P<0 \cdot 001$ ).

In Expt $2 \mathrm{~A}$, mean plasma melatonin concentration in the gilts receiving $0 \mathrm{mg}$ of melatonin was below the sensitivity of the assay throughout the period when the lights were on $(12: 00-18: 00 \mathrm{~h})$. After the lights were turned off, mean plasma melatonin concentration increased $(P<0.05)$ within $1 \mathrm{~h}$, and was still high at 22:00 h. In gilts fed pellets containing melatonin, mean plasma melatonin concentration was below the sensitivity of the assay before feeding, but it increased $(P<0.001)$ within $30 \mathrm{~min}$ of feeding, and was still high at 22:00 h. There was no significant difference in the mean plasma melatonin concentration in the post-feeding period among the gilts fed 1,2 or $4 \mathrm{mg}$ of melatonin. In Expt $2 \mathrm{~B}$, daily feeding of $1 \mathrm{mg}$ of melatonin increased $(P<0.05)$ the proportion of gilts which reached puberty. Among the 27 gilts which were fed melatonin, $15(55.6 \%)$ reached puberty compared with six of the $25(24 \%)$ control gilts.

These results show that, in contrast to constant-release melatonin implants, oral melatonin given each afternoon overcomes the seasonal inhibition of attainment of puberty in domestic gilts. These data provide further evidence that photoperiod is the environmental factor mediating seasonality in this species and support the conclusion that the diurnal rhythm of plasma melatonin, rather than the absolute concentration, 
is the important characteristic of melatonin secretion which pigs use to transduce photoperiodic information.

Keywords: melatonin; puberty; pig; season; gilt

\section{Introduction}

Work in our laboratory has established that photoperiod plays a major role in controlling the seasonal variation in attainment of puberty in domestic gilts (Paterson \& Pearce, 1990; Paterson et al., 1991), but how gilts monitor daylength has been the subject of debate. Recently, we have shown that domestic gilts display a diurnal variation in plasma melatonin concentration (Paterson et al., 1992) which is similar to that observed in other species that use melatonin for this purpose (Lincoln et al., 1982; Arendt, 1986). The pattern of plasma melatonin we observed in the dark phase, with peak concentrations measured $1-2 \mathrm{~h}$ after lights off followed by a gradual decline towards baseline, was almost identical to that reported for European wild pigs (Mauget et al., 1990). Reproductive activity in this species exhibits a high degree of seasonality, which is controlled by photoperiod (Mauget, 1982) and, as in other species, the diurnal variation in melatonin is believed to be the means by which photoperiodic information is transduced. The findings of Paterson et al. (1992) suggest that this physiological mechanism also operates in domestic pigs.

In sheep (Kennaway et al., 1982; Lincoln \& Ebling, 1985; English et al., 1986), deer (Adam \& Atkinson, 1984; Adam et al., 1989; Fisher et al., 1989) and goats (McGregor et al., 1989) inhibition of reproductive activity by long days can be overcome by the administration of exogenous melatonin, given either orally or by slow-release implant. We have already demonstrated that the seasorial inhibition of attainment of puberty in domestic gilts can be prevented by applying a short-day regimen (Paterson \& Pearce, 1990), but such methods may be difficult to use in combating seasonal reproductive dysfunctions at the practical level. Based on published work in other photoperiodic species, we hypothesized that administration of exogenous melatonin to domestic gilts would prevent the seasonal inhibition of puberty. In the experiments reported here, we studied the effect of exogenous melatonin, given either orally or by constant-release implant, on attainment of puberty and plasma melatonin concentrations in gilts which were expected to exhibit seasonal inhibition of puberty (Paterson et al., 1991).

\section{Materials and Methods}

\section{Animals, housing and treatments}

Two experiments were conducted using Large White $\times$ Landrace crossbred gilts from the herd at the West Australian Department of Agriculture Intensive Industries Research Centre, Medina.

Experiment 1: Constant-release melatonin implants. Forty-eight gilts born between 19 July and 4 August 1989 were housed in groups of eight in pens $3.2 \times 1.8 \mathrm{~m}$ (including $1.2 \times 1.8 \mathrm{~m}$ slatted area) in a fully enclosed grower shed with a capacity for 720 pigs. Allocation to pens was done when the gilts were moved from the weaner accommodation into the grower shed on 24 September (mean age $58 \pm 1.0$ days) and no further mixing or pen changes took place. The gilts remained isolated from boars throughout. The shed contained no mature boars and no male grower pigs over 100 days of age were housed within $25 \mathrm{~m}$ of the gilts. The gilts were fed ad libitum up to an average pen weight of $40 \mathrm{~kg}$. They were then restricted to $1.6 \mathrm{~kg}$ feed per pig per day, increasing each week by $0.1 \mathrm{~kg}$ up to a maximum of $2.4 \mathrm{~kg}$ feed per pig per day. In the ad libitum phase and during the first 4 weeks of restricted feeding, the diet contained $13.6 \mathrm{MJ} \mathrm{DE} / \mathrm{kg}$ and $19.0 \%$ crude protein with $1 \cdot 10 \%$ total lysine. Thereafter, the diet contained $13.0 \mathrm{MJ} \mathrm{DE} / \mathrm{kg}$ and $17 \cdot 1 \%$ crude protein with $1 \cdot 05 \%$ total lysine. The gilts were allocated to their pens according to litter of origin and body weight, so that litter mates were equally distributed between the two treatments which were: (i) control ( 3 pens, $n=24$ ), which received no melatonin implants and (ii) implanted ( 3 pens, $n=24$ ), which received two implants each containing $18 \mathrm{mg}$ of melatonin (Regulin, Schering Australia) subcutaneously behind the right ear at 126 days of age ( 1 December) and again at 161 days ( 5 January) and 196 days ( 9 February).

Blood samples $(5 \mathrm{ml})$ were obtained by venepuncture of a superficial vein on the left ear of eight gilts in each treatment on 1 December (126 days of age, before inserting the implants), 19 December (144 days of age) and 22 
January (178 days of age). Plasma was assayed for melatonin concentration by direct radioimmunoassay (RIA). All gilts were slaughtered on $8 \mathrm{March}$ (223 days) and their ovaries were recovered to determine their reproductive status. The presence of morphologically normal corpora lutea was the criterion for classification of a gilt as having reached puberty.

Experiment 2: Oral melatonin. In Part A, four gilts with a mean age of $125 \pm 1 \cdot 1$ days were housed in individual pens $(1.8 \times 1.2 \mathrm{~m})$ in an experimental facility containing 64 pens, but no other pigs. Lighting in the shed was supplied by natural daylength $(\sim 13 \cdot 6 \mathrm{~h} /$ day at the time of the bleed) supplemented with fluorescent lights such that the light intensity in the shed was $\sim 150$ lux. Shutters were closed and the lights turned off immediately after the 18:00 h blood samples were collected. The gilts were fed according to the feeding schedule outlined in Expt 1, except that their morning feed allocation was reduced by $200 \mathrm{~g}$ to allow for the afternoon feed of pellets. After 10 days of acclimatization to the pens and the feeding regimen the gilts were fitted with indwelling jugular vein catheters (Silastic medical grade tubing, i.d. $1.57 \mathrm{~mm}$, o.d. $3.17 \mathrm{~mm}$, Dow Corning Corp., Midland, MI, USA) under halothane anaesthesia. Commencing the next day ( 6 November 1990), each gilt received one of four doses of melatonin $(0,1,2$ or $4 \mathrm{mg}$ ) orally on each of four consecutive days such that all gilts received all doses, and all doses were represented on each day. Crystalline melatonin (Sigma Chemicals, St Louis, MO, USA) was dissolved in ethanol and $5 \mathrm{ml}$ of the appropriate concentration solution was absorbed onto $200 \mathrm{~g}$ of feed pellets. The ethanol was then allowed to evaporate overnight before feeding at 14:00 h the next day. On each of the 4 days, blood samples were collected via the catheters every 30 or $60 \mathrm{~min}$ from 12:00 to 22:00 h. Plasma was assayed for melatonin concentration by direct RIA.

In Part B, 52 gilts were moved into individual pens in the experimental facility at a mean age of $129 \pm 0.6$ days and mean weight of $64.9 \pm 0.78 \mathrm{~kg}$ on 3 December 1990 . For the first 7 days all gilts received $200 \mathrm{~g}$ of control pellets at 15:00 $\mathrm{h}$. The lighting and feeding regimen was the same as in Part A, except that the shutters were not closed in the evening. The gilts were allocated according to litter of origin and body weight, so that litter mates were equally distributed between the two treatments which were: (i) control $(n=25)$, which received pellets containing no melatonin and (ii) melatonin-treated $(n=27)$, which received $1 \mathrm{mg}$ of melatonin each day.

Treatments commenced on 10 December. Control pellets and pellets containing $1 \mathrm{mg}$ of melatonin, prepared as in Part A, were given at $15: 00 \mathrm{~h}$ each day until the day before slaughter on 7 March 1991 (221 \pm 0.6 days, $127.4 \pm 1.20 \mathrm{~kg}$ ). At slaughter the ovaries of all gilts were recovered to determine their reproductive status. The presence of morphologically normal corpora lutea was the criterion for classification of a gilt as having reached puberty.

\section{Melatonin assay}

Plasma melatonin was assayed by the direct RIA technique of Maxwell et al. (1989). This direct assay, which we have previously used to measure melatonin in pig plasma (Paterson et al., 1992), has been validated for sheep and deer plasma by high-performance liquid chromatography and electron capture, by gas chromatography mass spectroscopy and by comparison with an extraction-based assay which uses a different antiserum (Newman et al., in press). $\left[O\right.$-Methyl- $\left.{ }^{3} \mathrm{H}\right]$ melatonin $(85 \mathrm{Ci} / \mathrm{mol}$ ) was purchased from the Radiochemical Centre (Amersham, UK) and antiserum (Prospect 6A) to a prepared antigen, the hemisuccinimide derivative of melatonin conjugated to human serum albumin (Foldes et al., 1983), was raised in Merino ewes in our laboratory. All other reagents were commercially available reagent grade. Standard curves and quality controls were prepared in daytime plasma collected from gilts of a similar age in the Medina herd. Each assay included quality control standards as well as recovery standards obtained by analysis of porcine plasma with known amounts of exogenous (added) melatonin. The average limit of detection (two standard deviations below the mean of triplicate zero standards) for $1 \mathrm{l}$ assays in which samples from this experiment and the experiments described by Paterson et al. (1992) were assayed was $3.6 \mathrm{pg} / \mathrm{ml}$. The coefficient of variation of porcine samples was $10 \%$ at $80 \mathrm{pg} / \mathrm{ml}$ between assays and $13 \%$ within assays.

\section{Statistical analysis}

The data for age and weight were compared using Student's $t$ tests. The proportion of gilts which reached puberty on the two treatments in each experiment were compared using simple $2 \times 2$ Pearson $\chi^{2}$ tests. The plasma melatonin data in Expt 1 were subjected to an analysis of variance for repeated measures with treatment (control or implanted) as the main effect and sampling day as the repeated measure. The plasma melatonin data in Expt 2 were transformed logarithmically because the variance was proportional to the mean, and then subjected to an analysis of variance for repeated measures with treatment (dose of melatonin) as the main effect and sampling time as the repeated measure. Where analysis of variance revealed significant effects, comparisons of values between treatments were made on the transformed data using a least significant difference (LSD) based on the between-group s.e.m. calculated from a combination of the residual and treatment $\times$ pig mean squares as described by Cochran $\&$ Cox (1957), and comparisons between values within treatments were made using an LSD based on the within-group s.e.m. calculated from the residual mean square. To aid the reader, the melatonin data for Expt 2 are presented in Figs 3 and 4 as the back-transformed means, rather than on the log scale. All analyses were performed on an Apple Macintosh SE/30 microcomputer using programmes within the statistical package Statview $512+$, v 1.2 (Abacus Concepts Inc., Berkeley, CA, USA). 


\section{Results}

\section{Experiment 1: Constant-release melatonin implants}

The melatonin implants had no effect on the proportion of gilts which had reached puberty when they were slaughtered at 223 days of age. Among the 24 control gilts, two $(8 \cdot 3 \%)$ reached puberty compared with one of the $24(4 \cdot 2 \%)$ of the melatonin implanted gilts (Fig. 1). Analysis of variance revealed a significant effect of treatment $(P<0.001)$, day $(P<0.001)$ and treatment $\times$ day interaction $(P<0.001)$ on the mean plasma melatonin concentration on the three sampling days. Mean plasma melatonin concentration was below the sensitivity of the assay in the samples taken from the control gilts on all three days and in the samples taken from the implanted gilts prior to implantation at 126 days (Fig. 2). In the samples taken at 144 days, mean plasma melatonin concentrations were higher $(P<0.001)$ in the implanted than in the control gilts. In the samples taken at 178 days, 17 days after the insertion of the second pair of implants, the mean plasma melatonin concentration in the implanted gilts was again higher $(P<0.001)$ than that in the controls, and it was also higher $(P<0.001)$ than it had been at 144 days.

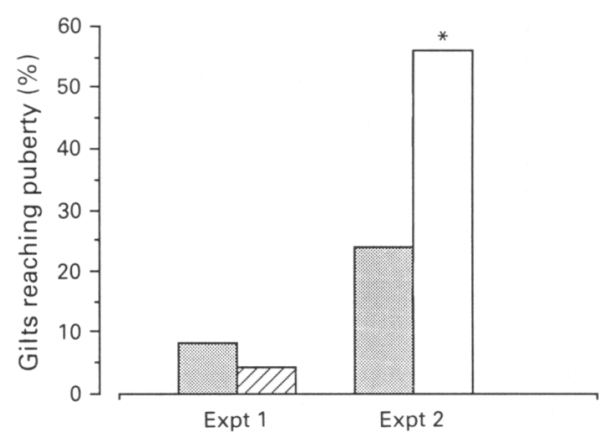

Fig. 1. Percentage of gilts reaching puberty among control gilts (圈), gilts given constant-release melatonin implants $(叉)$ and gilts fed $1 \mathrm{mg}$ of melatonin daily from 126 days of age $(\square)$; * difference between treatments at $P<0.05$.

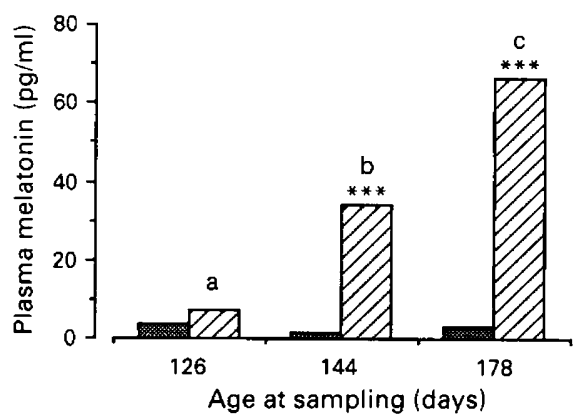

Fig. 2. Mean plasma melatonin concentrations in control gilts (圈) or gilts given constantrelease melatonin implants at 126,161 and 196 days of age $(\mathbb{Z})$ in Expt 1 . Within-treatment s.e.m. $=2.03,{ }^{* * *} P<0.001(\mathrm{LSD}=11.9)$; between-treatment s.e.m. $=2.68$, a vs. b vs. c $P<0.001(\mathrm{LSD}=15 \cdot 7)$. 


\section{Experiment 2: Oral melatonin}

In Part A, analysis of variance revealed a significant effect of treatment $(P<0.001)$, sample $(P<0.001)$ and treatment $\times$ sample interaction $(P<0.001)$ on mean plasma melatonin concentration in the serial samples. When mean plasma melatonin concentrations before feeding (period 1 , 12:00-14:00 h), after feeding while the lights were on (period 2, 14:30-18:00 h) and after dark (period 3, 18:30-22:00 h) were compared, analysis of variance revealed a significant effect of treatment $(P<0.01)$, period $(P<0.001)$ and treatment $\times$ period interaction $(P<0.001)$.

Gilts fed pellets not containing melatonin had mean plasma melatonin concentrations below the sensitivity of the assay throughout the period when the lights were on (12:00-18:00 h). One hour after the lights were turned off, mean plasma melatonin concentration had increased significantly $(0.43$ to $0.95 \mathrm{ng} / \mathrm{ml}$ on the $\log$ scale, within-treatment s.e.m. $=0.13, \mathrm{LSD}=0.45, P<0.05)$ and remained high until the end of sampling at 22:00 h. The back-transformed means for mean plasma melatonin concentration in gilts not fed melatonin are shown in Fig. 3a.

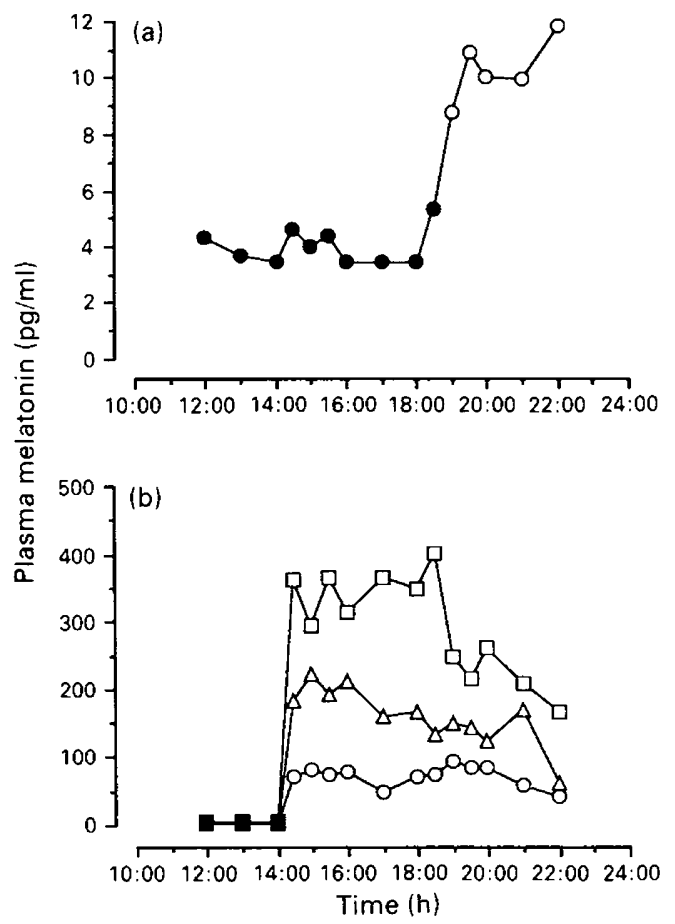

Fig. 3. Plasma melatonin concentrations (back-transformed means) in serial samples taken from 4 gilts receiving (a) control pellets or (b) pellets containing $1 \mathrm{mg}(\bullet), 2 \mathrm{mg}(\boldsymbol{\Delta})$ or $4 \mathrm{mg}$ (ם) of melatonin at 14:00 h, with lights out after the $18: 00 \mathrm{~h}$ sample. Values significantly $(0 \mathrm{mg}$, $P<0.05 ; 1,2,4 \mathrm{mg}, P<0.001)$ above the light-period baseline are indicated by open symbols.

In gilts fed pellets containing melatonin, mean plasma melatonin concentrations were below the sensitivity of the assay before feeding. By $30 \mathrm{~min}$ after feeding, mean plasma melatonin concentrations had increased significantly in all treatments $(1 \mathrm{mg}, 0.50$ to $1.86 ; 2 \mathrm{mg}, 0.46$ to $2.26 ; 4 \mathrm{mg}$, 0.88 to $2.56 \mathrm{ng} / \mathrm{ml}$ on the $\log$ scale, within-treatment s.e.m. $=0.13, \mathrm{LSD}=1 \cdot 10, P<0.001)$ and remained high until the end of sampling at 22:00 h. The differences between the treatments $1 \mathrm{~h}$ after feeding were not statistically significant ( 1.86 vs. 2.26 vs. $2.56 \mathrm{ng} / \mathrm{ml}$ on the log scale for the 1,2 and $4 \mathrm{mg}$ treatments, respectively; between-treatment s.e.m. $=0.22, \mathrm{LSD}=0.77$ for $P<0.05$ ). The back-transformed values for mean plasma melatonin concentrations in gilts fed pellets containing melatonin are shown in Fig. 3 b. 
Within each melatonin dose, mean plasma melatonin concentration increased between period 1 and period 2 ( $1 \mathrm{mg}, 0.56$ to $1.89 ; 2 \mathrm{mg}, 0.54$ to $2.29 ; 4 \mathrm{mg}, 0.85$ to $2.55 \mathrm{ng} / \mathrm{ml}$ on the log scale, within-treatment s.e.m. $=0.12, \mathrm{LSD}=1.05, P<0.001)$, but there were no statistically significant differences between period 2 and period 3 (1 mg, 1.89 vs. $1.89 ; 2 \mathrm{mg}, 2.29$ vs. $2.09 ; 4 \mathrm{mg}, 2.55$ vs. $2.37 \mathrm{ng} / \mathrm{ml}$ on the $\log$ scale, within-treatment s.e.m. $=0.12, \mathrm{LSD}=0.43$ for $P<0.05)$. The differences between the treatments in period 2 (1.89 vs. 2.29 vs. 2.55 for the 1,2 and $4 \mathrm{mg}$ treatments, respectively) and period 3 ( 1.89 vs. 2.09 vs. 2.37 for 1,2 and $4 \mathrm{mg}$ treatments, respectively) were not statistically significant (between-treatment s.e.m. $=0.20$, LSD $=0.70$ for $P<0.05$ ), although the difference between the $1 \mathrm{mg}$ and $4 \mathrm{mg}$ treatments in period 2 approached significance $(P=0.61)$. The back-transformed values for mean plasma melatonin concentrations for each treatment in each period are shown in Fig. 3 b.

In Part B, daily feeding of $1 \mathrm{mg}$ of melatonin increased $(P<0.05)$ the proportion of gilts which had reached puberty when they were slaughtered at 221 days of age. Among the 27 gilts which were fed melatonin, $15(55 \cdot 6 \%)$ reached puberty compared with six of the $25(24 \%)$ control gilts (Fig. 1).

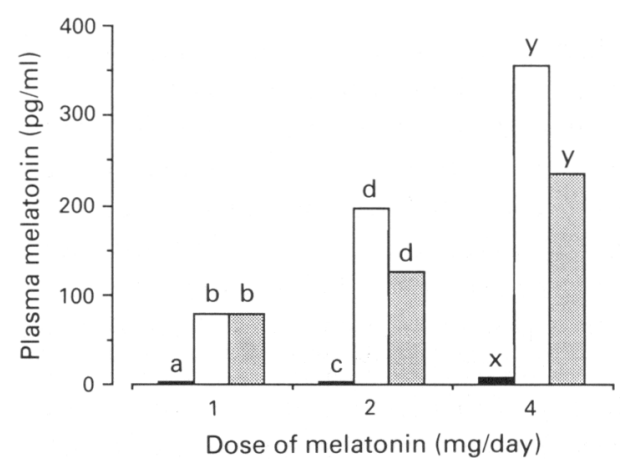

Fig. 4. Plasma melatonin concentrations (back-transformed means) in period 1 (12:00-14:00 h, 口), period $2(14: 30-18: 00 \mathrm{~h}, \square)$ and period $3(18: 30-22: 00 \mathrm{~h}$, 圈) in gilts receiving melatonin in feed pellets at 14:00 h. Within each dose, columns marked with different letters are significantly different $(P<0.001)$. No between-treatment comparisons reached statistical significance.

\section{Discussion}

The low percentage of control gilts which reached puberty in these experiments was in agreement with our previous studies in this herd (Paterson et al., 1989a, 1989b, 1991). The percentage which reached puberty in Expt 2 tended to be slightly higher than in Expt 1, but it was still much lower than we normally observe in uninhibited gilts. This trend was probably due to the gilts in Expt 2 being housed and fed individually, which resulted in faster growth and higher body weight than normally seen in group-housed gilts at the same age in this herd (Paterson et al., 1991). These data confirm that attainment of puberty is indeed inhibited by season in domestic gilts which reach 165 days of age in the summer months. Isolated control gilts therefore provide an excellent working model to test factors for their ability to overcome seasonal inhibition of reproductive processes in the pig, and it is against this background that the data for the melatonin-treated gilts is assessed.

The provision of constant-release melatonin implants to isolated gilts did not overcome the seasonal inhibition; the percentage of implanted gilts which reached puberty remained low. However, when exogenous melatonin was given orally, the percentage of gilts reaching puberty significantly increased, and reached the level normally seen when gilts either reach 165 days of age in the winter under shed conditions (Paterson et al., 1991) or are reared under an artificial short-day light 
regimen (Paterson \& Pearce, 1990). These results show clearly that oral application of melatonin to extend the period each day when plasma melatonin is increased, thus mimicking the natural endogenous diurnal rhythm typical of short days, overcomes the inhibition of puberty, but that the provision of a constant-release implant does not. In this, domestic pigs differ from sheep and deer, in which both implants and oral melatonin can stimulate reproductive activity under inhibitory photoperiods (Kennaway et al., 1982; Lincoln \& Ebling, 1985; Adam \& Atkinson, 1984; English et al., 1986; Adam et al., 1989). We have previously shown that constant-release melatonin implants given by the same regimen as used in Expt 1 provide a constant, high plasma melatonin concentration above which a diurnal rhythm cannot be detected (Paterson et al., 1992). It appears that gilts interpret this hormonal milieu as a total dark or ultrashort-day situation, conditions which are known to inhibit reproductive activity in pigs (Hacker et al., 1979; Ntunde et al., 1979; Diekman \& Greiger, 1988). In sheep, melatonin implants increase the daytime baseline concentration of plasma melatonin, but they do not abolish the diurnal rhythm (Foldes et al., 1990). This suggests that, in sheep, both the baseline and the diurnal rhythm of melatonin are important, but in pigs it appears that the diurnal rhythm is the criterion by which information about daylength is transduced.

The plasma melatonin data collected in these experiments are important for a number of reasons. First, they confirm our previous report (Paterson et al., 1992) that there is a diurnal rhythm of plasma melatonin in domestic pigs, and extend that finding to animals housed under natural, rather than artificial light. It must now be accepted that the phenomenon of a diurnal rhythm of plasma melatonin does indeed exist in domestic pigs, and the controversy that has surrounded this question is now resolved. Failure in previous studies to observe a diurnal rhythm in plasma melatonin (McConnell \& Ellendorff, 1987; Diekman et al., 1990; Peacock et al., 1990a, b) may now be attributed to inadequacies in assay methodology or anomalies in the experimental conditions under which the samples were collected.

The second important finding of this study is that melatonin is orally active in domestic pigs. Plasma melatonin was increased within $30 \mathrm{~min}$ of ingestion of pellets containing melatonin and it remained high for at least $8 \mathrm{~h}$. Plasma concentrations were declining by 22:00 h, and it appears likely that they would have returned to basal levels by the morning, although we do not have any data between $22: 00 \mathrm{~h}$ and 12:00 h the next day to substantiate this. To our knowledge, there are no published data from serial blood samples in pigs fed melatonin to compare with our results. In humans, Wetterberg et al. (1977) reported a transitory peak in plasma concentrations following feeding of melatonin, but in sheep and goats oral administration of melatonin increased plasma concentrations for at least $7 \mathrm{~h}$ (Kennaway \& Seamark, 1980). These authors suggested that the different plasma pattern in humans and sheep was due to the retention of melatonin in the rumen and subsequent absorption over a longer period further down the gastrointestinal tract. Our data for pigs are similar to those of Kennaway \& Seamark (1980) for sheep and goats and bear little resemblance to the data for humans, despite the fact that pigs are monogastrics like humans. Either absorption of melatonin is much slower in pigs than in humans or the doses used in the present study were so large that the absorption phase was prolonged. The data support the latter suggestion, because melatonin appeared in the plasma rapidly after feeding, but then plasma concentrations remained high before starting to decline some $6 \mathrm{~h}$ later. This period of stability can be interpreted to mean that absorption of melatonin from the gut was continuing at about the same rate as clearance from the plasma was taking place in this period. The fact that the mean plasma concentrations measured in our pigs after feeding were over 100 times greater than the basal concentrations and over 10 times greater than the nocturnal increase in control gilts, even at the lowest dose of melatonin, further supports the suggestion that the doses used may have led to a lengthy absorption phase.

Although we have previously measured high plasma concentrations in implanted gilts (Paterson et al., 1992), the effective life of melatonin implants in pigs has not previously been reported. Plasma melatonin concentrations in the implanted gilts were increased 18 days after receiving the 
first pair of implants. In the samples taken 17 days after receiving the second pair of implants, plasma melatonin concentrations were double those measured at the first sampling 34 days previously, indicating that the first pair of implants were still releasing melatonin at about the same rate and that a simple additive effect was taking place. This shows that the effective life of these melatonin implants was at least 51 days, and may in fact have been much longer. Foldes $e t$ al. (1990) have reported that similar constant-release implants to those we used have an effective life of $\sim 90$ days in sheep.

The plasma melatonin concentrations measured in the implanted pigs were lower than those in the pigs which received melatonin in their feed, and it could be suggested that the ineffectiveness of the implants was due to these lower concentrations. However, we have previously measured mean plasma melatonin concentrations of $>90 \mathrm{pg} / \mathrm{ml}$ in gilts treated with the same implant regimen (Paterson et al., 1992) and these values are comparable with those measured in pigs fed $1 \mathrm{mg}$ of melatonin in the present experiment. It therefore appears likely that the difference in the pubertal response to implants and oral melatonin was due to the diurnal pattern provided by the oral application rather than to the absolute plasma concentrations achieved. The difference in the plasma melatonin concentrations measured in the implanted animals in our two studies may be explained by the site from which samples were taken. In the experiment reported in Paterson et al. (1992), we collected blood from indwelling catheters placed in the right jugular vein, whereas in the present experiment we collected blood from a superficial vein in the left ear. Since the implants were placed behind the right ear in both experiments, it is not surprising that higher concentrations of plasma melatonin were measured in the samples collected from the ipsilateral jugular vein, which would have been carrying blood draining directly from the area containing the implants. This observation points out the necessity for care to be taken to standardize the site of blood sampling within each experiment and to define the sampling site when comparisons are drawn among experiments.

In conclusion, the main finding of these experiments, that oral melatonin given each afternoon overcomes the seasonal inhibition of attainment of puberty in domestic gilts, provides further evidence that photoperiod is the environmental factor mediating seasonality in this species. The data on plasma melatonin described here, together with the finding that constant-release melatonin implants did not affect puberty, support the conclusion that the diurnal rhythm of plasma melatonin, rather than the absolute concentration is the important characteristic of melatonin secretion which pigs use to transduce photoperiodic information. These findings open the way to investigate the use of oral melatonin in other situations in which reproduction in pigs is adversely affected by season.

The dedicated technical assistance of H. G. Payne and J. Hooper is gratefully acknowledged, as is the assistance of M. F. D'Antuono with the statistical analyses. The contribution of R. M. Hoskinson and R. Rigby to the preparation of the melatonin antigen on which the melatonin assay is based is also gratefully acknowledged. Schering Australia Pty Ltd generously donated the melatonin implants used in Expt 1.

\section{References}

Adam, C.L. \& Atkinson, T. (1984) Effect of feeding melatonin to red deer (Cervus elaphus) on the onset of the breeding season. J. Reprod. Fert. 72, 463-466.

Adam, C.L., Moir, C.E. \& Shiach, P. (1989) Melatonin can induce year-round cyclicity in red deer (Cervus elaphus). J. Reprod. Fert. 87, 401-408.

Arendt, J. (1986) Role of the pineal gland and melatonin in seasonal reproductive function in mammals. Oxford Reviews of Reproductive Biology 8, 266-320.
Cochran, W.G. \& Cox, G.M. (1957) Experimental Designs, 2nd edn, p. 298. John Wiley and Sons Inc., New York.

Diekman, M.A. \& Greiger, D.M. (1988) Influence of various intensities of supplemental lighting during decreasing daylengths on puberty in gilts. Anim. Reprod. Sci. 16, 295-301.

Diekman, M.A., Green, M.L. \& Hudgens, R.E. (1990) Serum concentrations of melatonin in gilts exposed Downloaded from Bioscientifica.com at 04/26/2023 02:15:48PM 
to total darkness or direct sunlight. J. Reprod. Fert. Suppl. 40, 371 (abst).

English, J., Poulton, A.L., Arendt, J. \& Symons, A.M. (1986) A comparison of the efficiency of melatonin treatments in advancing oestrus in ewes. $J$. Reprod. Fert. 77, 321-327.

Fisher, M.W., Fennessy, P.F. \& Johnstone, P.D. (1989) The timing of melatonin treatment affects the seasonal onset of ovarian activity, coat growth and live weight in young red deer hinds. Proc. Aust. Soc. Reprod. Biol. 21, 130 (abst).

Foldes, A., Hoskinson, R.M., Scaramuzzi, R.J. \& Maxwell, C.A. (1983) Modification of sheep pineal $\beta$-adrenoceptors by some gonadal steroids but not by melatonin. Neuroendocrinology 37, 378-385.

Foldes, A., Maxwell, C.A., Rintoul, A.J., Scaramuzzi, R.J. \& Baker, P. (1990) Effects of melatonin implants on selected hormonal profiles and wool growth in merino wethers. Animal Production in Australia 18, $208-211$.

Hacker, R.R., King, G.J., Ntunde, B.N. \& Narendran, R. (1979) Plasma oestrogen, progesterone and other reproductive responses of gilts to photoperiods. $J$. Reprod. Fert. 57, 447-451.

Kennaway, D.J. \& Seamark, R.F. (1980) Circulating levels of melatonin following its oral administration or subcutaneous injection in sheep and goats. Aust. $J$. Biol. Sci. 33, 349-353.

Kennaway, D.J., Gilmore, T.A. \& Seamark, R.F. (1982) Effects of melatonin feeding on serum prolactin and gonadotrophin levels and the onset of seasonal estrous cyclicity in sheep. Endocrinology 110, $1766-1772$.

Lincoln, G.A. \& Ebling, F.J.P. (1985) Effect of constantrelease implants of melatonin on seasonal cycles in reproduction, prolactin secretion and moulting in rams. J. Reprod. Fert. 73, 241-253.

Lincoln, G.A., Almeida, O.F.X., Klandorf, H. \& Cunningham, R.A. (1982) Hourly fluctuations in the blood levels of melatonin, prolactin, luteinizing hormone, testosterone, tri-iodothyronine, thyroxine and cortisol in rams under artificial photoperiods and the effects of cranial sympathectomy. $J$. Endocr. 92, 237-250.

Mauget, R. (1982) Seasonality of reproduction in the wild boar. In Control of Pig Reproduction, pp. 509-526. Eds D. J. A. Cole \& G. R. Foxcroft. Butterworths, London.

Mauget, R., Ravault, J.P., Chesneau, D., Locatelli, A., Saboureau, M., Sempere, A.J. \& Lacroix, A. (1990) Peripheral melatonin concentrations in the European wild boar: circadian and seasonal variations. $J$. Reprod. Fert. Suppl. 40, 371 (abst).

Maxwell, C.A., Rintoul, A.J., Foldes, A., Downing, J.A., Scaramuzzi, R.J. \& Carter, N.B. (1989) Seasonal modification of ovine pineal function. 2. Steroidal effects on melatonin and prolactin profiles. Neuroendocrinology 50, 274-279.

McConnell, S.J. \& Ellendorff, F. (1987) Absence of nocturnal melatonin surge under long and short artificial photoperiods in the domestic sow. J. Pineal Res. 4, 201-210 and Erratum 4, 341-348.

McGregor, B.A., McPhee, S.R., Williams, A.W., Earle, C.R. \& Staples, L.D. (1989) Melatonin treatment improves kidding percentage in angora does. Proc. Aust. Soc. Reprod. Biol. 21, 98 (abst).

Newman, R.E., Foldes, A., Maxwell, C.A., Rigby, R.D.G. \& Wynn, P.C. (in press) Identification of a seasonal elevation in daytime melatonin levels associated with the rut in fallow bucks (Dama dama): the effect of daylength and exogenous melatonin. J. Pineal Res.

Ntunde, B.N., Hacker, R.R. \& King, G.J. (1979) Influence of photoperiod on growth, puberty and plasma LH levels in gilts. J. Anim. Sci. 48, 1401-1406.

Paterson, A.M. \& Pearce, G.P. (1990) Attainment of puberty in domestic gilts reared under long-day or short-day artificial light regimens. Anim. Reprod. Sci. 23, $135-144$.

Paterson, A.M., Hughes, P.E. \& Pearce, G.P. (1989a) The effect of limiting the number of days of contact with boars, season and herd of origin on attainment of puberty in gilts. Anim. Reprod. Sci. 18, 293-301.

Paterson, A.M., Hughes, P.E. \& Pearce, G.P. (1989b) The effect of season, frequency and duration of contact with boars on the attainment of puberty in gilts. Anim. Reprod. Sci. 21, 115-124.

Paterson, A.M., Pearce, G.P. \& D'Antuono, M.F. (1991) Seasonal variation in attainment of puberty in isolated and boar-exposed domestic gilts. Anim. Reprod. Sci. 24, 323 333.

Paterson, A.M., Martin, G.B., Foldes, A., Maxwell, C.A. \& Pearce, G.P. (1992) Concentrations of plasma melatonin and luteinizing hormone in domestic gilts reared under artificial long or short days, $J$, Reprod. Fert. 94, 85-95.

Peacock, A.J., Love, R.J. \& Evans, G. (1990a) Plasma melatonin levels in sows are lowest during winter. $J$. Reprod. Fert. Suppl. 40, 371 (abst).

Peacock, A.J., Love, R.J. \& Evans, G. (1990b) Photoperiodic involvement in elevated $\mathrm{LH}$ concentrations in sows during summer. J. Reprod. Fert. Suppl. 40, 371 (abst).

Wetterberg, L., Erikson, O., Friberg, Y. \& Vangbo, B. (1977) A simplified radioimmunoassay for melatonin and its application to biological fuids. Preliminary observations on the half-life of plasma melatonin in man. Clin. Chim. Acta 86, 169-177.

Received 14 June 1991 